\title{
Random field formulation of composite laminates
}

\author{
Wen-Fang Wu *, Ho-Chieh Cheng, Chu-Kun Kang \\ Department of Mechanical Engineering, National Taiwan University, Taipei 10617, Taiwan, ROC
}

\begin{abstract}
A numerical simulation algorithm is developed in the present paper in order to characterize the random strength distribution of a batch of composite laminates. The program is based on the assumption that the axial, transverse and shear strengths of the composite lamina are statistically characterized by two-dimensional random fields. Monte Carlo method is employed to generate these strengths. When loading is applied to the composite laminate, the resultant stresses are then compared with the generated strengths of the lamina based on Tsai-Hill or Tsai-Wu criterion. The strength of the laminate is then determined. Owing to the random strength distributions of the lamina, the strength of the composite laminate is also statistically distributed which can provide useful information on the reliability assessment of a structure or component made of the laminate. In order to verify the above simulation algorithm, experimental work is conducted in the present study. It is found that the proposed simulation algorithm can adequately capture the behavior of the experimental result. It is also concluded that, for the strength of the studied laminates, both the numerical simulation and experimental results can be fitted well by the normal probability density function. (C) 2000 Elsevier Science Ltd. All rights reserved.
\end{abstract}

Keywords: Random field; Reliability; Composite laminates; Tensile strength; Simulation

\section{Introduction}

Owing to inherit complications in the manufacturing process, the mechanical properties of composite materials usually display more inherent scatter than those of the metallic materials. The anisotropic nature of fiberreinforced composites makes the scatter even more significant. The result is that, although many macro- or micro-mechanics-based failure criteria have been derived and proposed to predict the strength of the materials, no prediction formula has been proved to be completely exact. To overcome this difficulty, probability, reliability and statistical theories have been introduced in the analysis of composite materials [1-8]. An analysis mixed probability or statistical theory with the traditional mechanics analysis results in not only the average but also the deviation trend of what can usually be provided by a deterministic composite mechanics analysis. Through the analysis, different values of the material strength may be obtained which resemble the scatter nature of the experimental results. Further application of probability or statistical analysis to these data offers us a basis for the safety and reliability as-

${ }^{*}$ Corresponding author. sessment of structures made of composite materials [5-8].

The concept of random field has been used extensively in the structural reliability analysis [9]. It can also be applied to the analysis of composite strength $[1,10]$. The simplest one-dimensional random field related to the composite strength analysis may be the famous 'weakest link' theory. Application of the theory results in a Weibull distribution of the material strength. The Weibull distribution is, in fact, a certain kind of asymptotic distribution of the extremes [11]. It, however, has been pointed out to be too conservative in the estimation of structure failure probability [1]. More extensive studies were therefore suggested [1].

One way to extend the one-dimensional weakest link theory is the introduction of a two-dimensional random field. In fact, it has been suggested that a two-dimensional random field may be an appropriate model to characterize the local strength or stiffness variation within a composite plate [10]. If the random field formulation can be justified, then many mathematical formulas derived in the field of structural safety can be employed in the probability or reliability analysis of composite materials. Based on this consideration, the major purpose of the present study is to examine whether the random filed formulation can really be used 
to characterize the material strength of the composite material. To begin with a simple case, a composite laminate subjected to unidirectional tension is considered. Since the strength of a composite laminate is related to the layout of the composite layers and its prediction usually depends on the selection of a failure criterion, both of the layout and failure criteria have to be considered in the present study. To verify whether the proposed formulation is appropriate, experimental work has been performed. Both of the simulation and experimental results will be reported in the present paper.

\section{Composite mechanics and random fields}

The mechanics analysis of composite laminates has been well developed and documented in standard textbooks such as Refs. $[12,13]$. In particular, for a composite laminate subjected to a unidirectional load, the strain vector of the laminate can be obtained first. It can then be used to find the stress vector for each layer of the laminate. The principal stresses of the layer can also be obtained accordingly. Having the knowledge of the stress vector, one can predict whether a lamina will fail or not based on an appropriate failure criterion. Among various failure criteria proposed by many researchers, the following two well-known criteria are employed in the present study. The first one is the Tsai-Hill failure criterion which states that failure occurs when [12-14]

$\frac{\sigma_{1}^{2}}{X^{2}}-\frac{\sigma_{1} \sigma_{2}}{X^{2}}+\frac{\sigma_{2}^{2}}{Y^{2}}+\frac{\tau_{12}^{2}}{S^{2}} \geqslant 1$,

in which $\sigma_{1}, \sigma_{2}$ and $\tau_{12}$ are normal and shear stresses, $X$ the longitudinal strength, $Y$ the transverse strength and $S$ is the shear strength of the composite lamina. All are related to the principal directions. The second failure criterion is the Tsai-Wu criterion which states that failure occurs when

$$
\begin{aligned}
& \left(\frac{1}{X_{\mathrm{t}}}+\frac{1}{X_{\mathrm{c}}}\right) \sigma_{1}+\left(\frac{1}{Y_{\mathrm{t}}}+\frac{1}{Y_{\mathrm{c}}}\right) \sigma_{2}-\frac{\sigma_{1}^{2}}{X_{\mathrm{t}} X_{\mathrm{c}}}-\frac{\sigma_{2}^{2}}{Y_{\mathrm{t}} Y_{\mathrm{c}}}+\frac{\tau_{12}^{2}}{S^{2}} \\
& -\sigma_{1} \sigma_{2} \sqrt{\frac{1}{X_{\mathrm{t}} X_{\mathrm{c}} Y_{\mathrm{t}} Y_{\mathrm{c}}}} \geqslant 1,
\end{aligned}
$$

where the difference between the tensile strength and compression strength are considered. They are denoted, respectively, by the subscripts $t$ and $c$. It should be noted that the last term at the left-hand side of the above equation comes from an equivalent replacement of a fourth-order strength tensor $[13,15]$.

Recognizing the fact that local strengths of composite materials may vary from one location to the other, a random field may be employed to characterize the strengths together with their variation. If composite laminates are considered, a two-dimensional random field is an appropriate choice for the mathematical modeling of the strength. In the present study, the strength of the supplier-provided composite laminae is assumed to be a two-dimensional random field. Owing to the strength variation of the laminae, the strength of the laminates, when subjected to certain loads, also vary from one laminate to the other. For a batch of laminates having the same dimensions and treatment conditions, the failure location may also occur randomly.

As stated above, the strengths of the composite laminae are modeled as two-dimensional random fields in the present study. In particular, the longitudinal strength of the laminae is considered to be

$X(x, y)=\bar{X}[1+f(x, y)]$.

The transverse strength of the laminae is

$Y(x, y)=\bar{Y}[1+g(x, y)]$,

and the shear strength is modeled as

$S(x, y)=\bar{S}[1+h(x, y)]$.

In the above equations, an over-bar indicates the mean value of the considered variable; $f, g$ and $h$ are two-dimensional homogeneous random fields. The subscripts $t$ and $\mathrm{c}$ may be added to variables in Eqs. (3) and (4) if the Tsai-Wu criterion is considered. A minus sign also has to be added to the generated values to indicate compression. Without loss of generality, all random fields are assumed to have the same form of power spectral density as

$\Phi\left(\kappa_{x}, \kappa_{y}\right)=\frac{d_{x} d_{y} V}{4 \pi}\left[-\frac{1}{2}\left(d_{x}^{2} \kappa_{x}^{2}+d_{y}^{2} \kappa_{y}^{2}\right)\right]$,

where $V$ is the variance of the random field; $\kappa_{x}$ and $\kappa_{y}$ the wave numbers obtained from the Fourier transformation of the spatial distances in the longitudinal and transverse directions respectively; $d_{x}$ and $d_{y}$ are measures of correlation length in the respective directions.

To generate sample functions from a given power spectral density as that described in Eq. (6), the following formula can be used [16]:

$$
\begin{aligned}
f(x, y)= & \sqrt{2} \sum_{i=1}^{M_{1}} \sum_{j=1}^{M_{2}} \sqrt{2 \Phi\left(\kappa_{1 i}, \kappa_{2 j}\right) \Delta \kappa_{1} \Delta \kappa_{2}}\left[\operatorname { c o s } \left(\kappa_{1 i} x\right.\right. \\
& \left.\left.+\kappa_{2 j} y+\phi_{i j}^{1}\right)+\cos \left(\kappa_{1 i} x+\kappa_{2 j} y+\phi_{i j}^{2}\right)\right]
\end{aligned}
$$

where

$\Delta \kappa_{1}=\frac{\kappa_{1 u}}{M_{1}}, \quad \Delta \kappa_{2}=\frac{\kappa_{2 u}}{M_{2}}$,

$\kappa_{1 i}=i \Delta \kappa_{1}, \quad \kappa_{2 j}=j \Delta \kappa_{2}$.

In the above equations, $\kappa_{1 u}$ and $\kappa_{2 u}$ are the upper cutoff wave numbers corresponding to directions $x$ and $y$ respectively, $M_{1}$ and $M_{2}$ are the number of intervals in the discretization of the spectrum along directions $x$ and 
$y$ respectively, and $\phi_{i j}^{1}$ and $\phi_{i j}^{2}$ are random phase angles independently and uniformly distributed between 0 and $2 \pi$. Although Eq. (7) has the appropriate notations for the digital generation of sample functions of stochastic field $f(x, y)$, it can be equally well used for $g(x, y)$ and $h(x, y)$ with obvious changes of notation.

\section{Simulation for laminate strength}

According to concepts introduced in the previous sections, a computer simulation program can be established to find the strengths of a batch of composite laminates. Although the laminates have the same geometric composition and are subjected to the same loading condition, their strengths will be found differently due to the above random field consideration. To be concise, the procedure for the computer simulation program is as follows.

1. Input the mechanical properties of the lamina. Aside from the supplier-provided data, some fundamental tests may be needed. In particular, for the random field modeling of lamina strength, the mean value and standard deviation of the strength must be known in advance. As for other parameters such as $d_{x}$ and $d_{y}$, appropriate values are assumed at this stage.

2. Generate random fields to resemble the strengths of the lamina.

3. According to its layer construction, find the stiffness matrix of the laminate.

4. If the laminate is subjected to a unidirectional load and the load is uniformly distributed to result in a nominal stress $\sigma$, the strains of the laminate can then be found from standard composite analysis. In the simulation program, a reasonably smaller value of $\sigma$ may be considered as the starting point for finding the laminate strength.

5. Again, from standard composite analysis, the stresses and principal stresses of the lamina can be found.

6. Putting the results of steps 2 and 5 into the Tsai-Hill or Tsai-Wu failure criterion to check whether each layer of the composite laminate will fail locally or not.

7. If no failure occurs, reasonably increase the value of $\sigma$ and repeat the steps of 5 and 6.

8. If failure occurs at some location of a certain lamina, one can eliminate the lamina from the laminate by assigning zero properties to the lamina and then repeat the steps of 3,5,6 and 7. Based on previous experiences, this step can sometimes be ignored for angleply composites since many composite failures are attributed to the first-ply failure and no 'knee' result is found $[12,14]$.

9. Under all circumstances, the maximum nominal stress that the composite laminate can sustain is named the strength of the laminate.
10. Repeat the above procedure for another laminate selected from the same batch of composite plates.

The flow chart shown in Fig. 1 summarizes the above analytical and simulation procedure.

\section{Experiment}

Experimental work has been carried out to find the strengths of laminates arranged in differently oriented layers. In addition to the average values, standard deviations of the strengths are also examined. To begin with the experiment, composite laminates have to be made out of the purchased prepreg taps. The prepreg is Type P305 T300 Graphite/Epoxy made by Torayca, which has a fiber content of $63 \%$ of the total weight. Arranged in different fiber orientations, the prepreg taps were first cut into square sheets of $23 \times 23 \mathrm{~cm}^{2}$ in size, and then stacked together according to what is needed. The stacked composites were then cured under a specific pressure/temperature condition suggested by the manufacturer to form the desired laminates.

In our experiment, all the composite laminates consist of 16 prepreg layers. Some laminates have their prepreg fibers arranged all in the same direction and some are particularly arranged to produce the $[0 / 45 / 90 /-45]_{2 s}$ laminates that were extensively studied in the present as

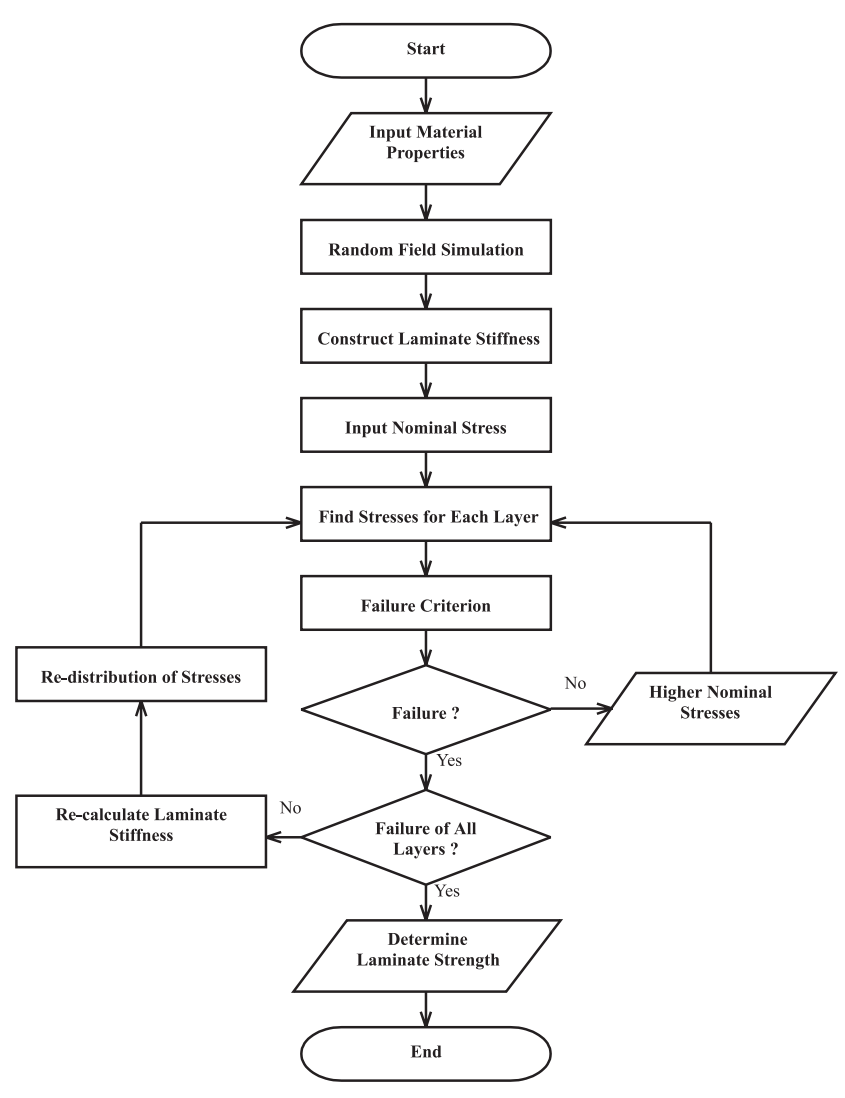

Fig. 1. Simulation flow chart. 
well as other related projects. Based on ASTM D3039 standard, the laminates were cut into specimens which have the dimensions shown in Fig. 2. An MTS 810 machine was then used to perform the unidirectional tensile tests. The displacement-controlled load was applied at an extension rate of $0.1 \mathrm{~mm} / \mathrm{s}$. During the experimental process, the applied load and the gauge displacement were closely monitored and recorded.

The result of the experiment is summarized in Table 1, which includes the mean values and standard deviations of the laminates of different groups. For each group, ten to fifteen specimens were tested. The results for those unidirectional stacked laminates can also be plotted in a curve shown in Fig. 3. From monitoring the load and displacement curves, the average Young's moduli for those laminates stacked at the unidirectional angles of $0^{\circ}$ and $90^{\circ}$ are found to be $126.00 \mathrm{GPa}$ and $292.54 \mathrm{MPa}$, respectively. Their corresponding standard deviations are $12.51 \mathrm{GPa}$ and $4.32 \mathrm{MPa}$ respectively. The loaddisplacement curve for each of the fifteen tested specimens of the $[0 / 45 / 90 /-45]_{2 s}$ laminates is plotted in Fig. 4. It seems that the knees are not very obvious for this experimental data, which is similar to the experimental result shown in Halpin's book [17].
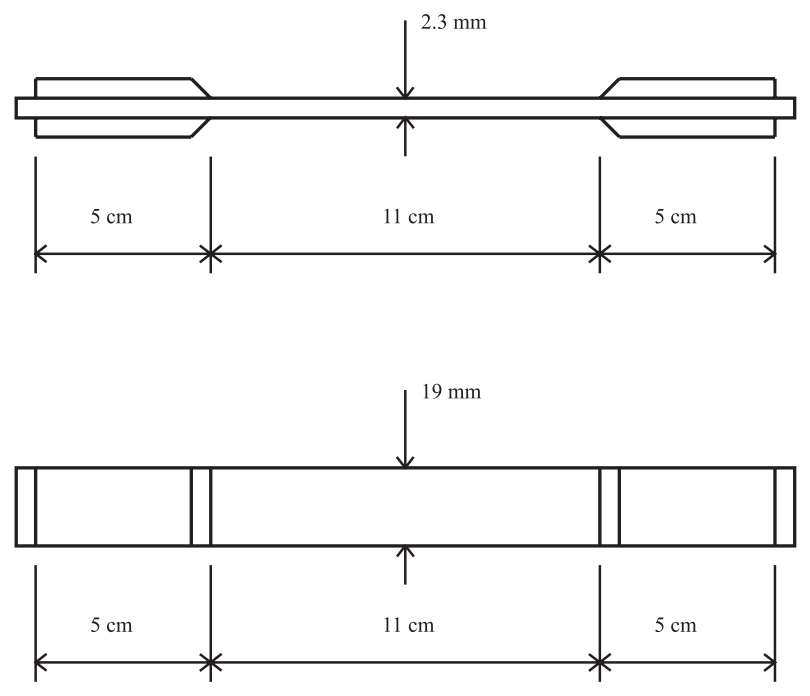

Fig. 2. The specimen.

Table 1

Experimental result

\begin{tabular}{lcc}
\hline Angle (degree) & $\begin{array}{l}\text { Mean strength } \\
(\mathrm{MPa})\end{array}$ & $\begin{array}{l}\text { Standard } \\
\text { deviation }(\mathrm{MPa})\end{array}$ \\
\hline 0 & 1469.86 & 46.70 \\
15 & 292.54 & 4.32 \\
30 & 147.64 & 2.93 \\
45 & 102.36 & 2.33 \\
60 & 84.78 & 1.96 \\
90 & 74.90 & 2.24 \\
{$[0 / 45 / 90 /-45]_{2 S}$} & 481.01 & 37.03 \\
\hline
\end{tabular}

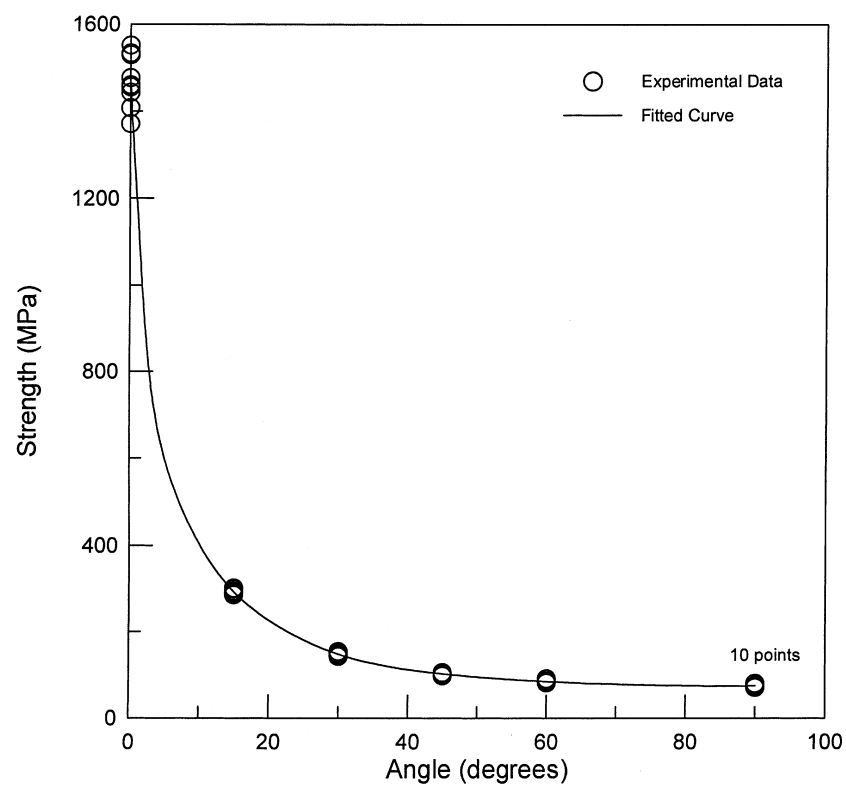

Fig. 3. Strengths for unidirectional stacked laminates.

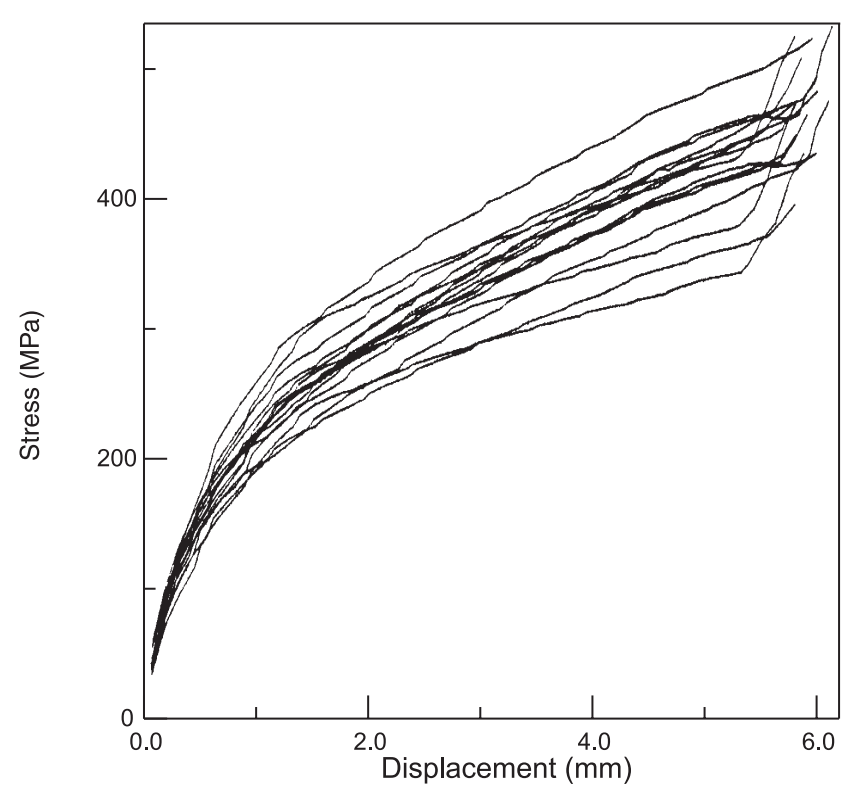

Fig. 4. Load-displacement curves for $[0 / 45 / 90-45]_{2 s}$ laminates.

\section{Verification of simulation algorithm}

Aside from the above experimental work, numerical simulation has also been carried out in the present study. In particular, the axial strength and shear strength of the lamina are all assumed to be two-dimensional random fields as introduced previously. The following values are used for the simulation: $\bar{X}_{\mathrm{t}}=$ $1469.86 \mathrm{MPa}, \bar{Y}_{\mathrm{t}}=74.90 \mathrm{MPa}, \bar{X}_{\mathrm{c}}=1142.57 \mathrm{MPa}, \bar{Y}_{\mathrm{c}}=$ $212.50 \mathrm{MPa}, \bar{S}=80.90 \mathrm{MPa}$, and $d_{x}=d_{y}=1.00 \mathrm{~cm}$, in which the average strengths are obtained experimentally and the correlation lengths are made by reasonable as- 


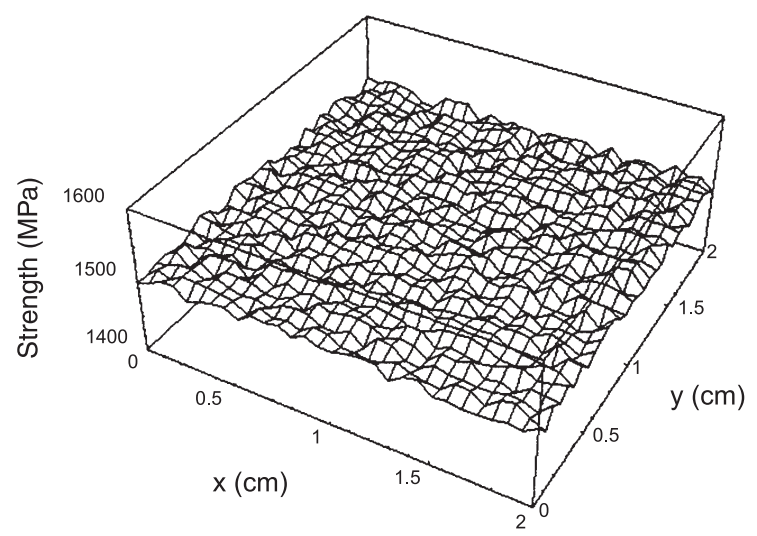

Fig. 5. Variation of the lamina strength by simulation.

sumption. The physical meaning of the correlation length here is that it characterizes the correlation between the strength of the elemental lamina and those of its neighboring elements. A higher correlation length indicates that the lamina strength is less influenced by its neighboring elements as compared to that of a lower correlation length. In the simulation process, the variances of the lamina strengths are also obtained experimentally, and some of these values are shown in Table 1. The simulated random field can be seen from figures such as the one shown in Fig. 5, which indicates the strength variation of the lamina.

The generated random fields representing the strengths of the laminae are then used in conjunction with the mechanics of the composite laminates introduced previously in order to find the possible local failure as well as global failure of an arbitrarily selected composite laminate. The simulation procedure follows those steps shown in Fig. 1 and the results are summarized in Table 2 in which both the unidirectional and the $[0 / 45 / 90 /-45]_{2 s}$ angle-ply laminates are included. Twenty samples are considered for each case of the simulation. It is found that the simulation results are very compatible with those experimental results shown in Table 1. It is also found that, for some cases, the Tsai-Wu failure criterion is found to be more appropriate, while for other cases, the Tsai-Hill criterion prevails.
To study the reliability of the composite laminates, both the experimental results and the simulation results are plotted in normal, log-normal and Weibull probability papers, and curve fitting is performed. Chi-square test is then used to test the goodness-of-fit [18]. It is found that normal or log-normal probability distribution fits the experimental as well as the simulation result better than the Weibull distribution. Typical results are shown in Figs. 6 and 7 in which the former is referred to the case of $[0 / 45 / 90 /-45]_{2 s}$ angle-ply laminates and the latter is referred to the unidirectional laminates extended at a non-principal angle of $60^{\circ}$. After the curve fitting and statistical test procedure, a reliability curve as that shown in Fig. 8 can be plotted for each case of the studied composite laminates. The curve can be used in the reliability design of a structure or mechanical component made of the studied composite laminates.

\section{Concluding remarks}

The major purpose of the present study is to introduce a probabilistic model to reflect the spatial strength variation of the composite materials. The principal idea lies in the interpretation that the material strength can be idealized as a multi-dimensional stochastic field. In this way, Monte Carlo simulation can be employed in conjunction with the well developed composite mechanics analysis to find the mean as well as deviation of the material properties. Composite laminates accompanied with a two-dimensional random field modeling are selected for a preliminary investigation of the above concept. Both experimental testing and the numerical simulation have been carried out in the present study. By comparing the experimental and simulation results, it is concluded that the proposed numerical simulation algorithm can adequately predict the experimental results. Under these circumstances, tedious experimental work may be reduced to the minimum. Some experimental tests may be replaced by simulation work in establishing a reliability model for composite materials. The model can then be used in the mechanical design of composite structures. For the present study, it is found that a normal probability distribution can appropriately

Table 2

Simulation result

\begin{tabular}{llccc}
\hline Angle (degree) & Tsai-Hill & & Tsai-Wu & \\
\cline { 2 - 3 } & Mean $(\mathrm{MPa})$ & Std. $(\mathrm{MPa})$ & Mean $(\mathrm{MPa})$ & Std. $(\mathrm{MPa})$ \\
\hline 0 & 1471.90 & 42.76 & 284.86 & 41.06 \\
15 & 285.93 & 4.35 & 147.15 & 7.67 \\
30 & 150.85 & 2.89 & 102.14 & 2.80 \\
45 & 106.68 & 3.19 & 85.16 & 3.18 \\
60 & 87.09 & 3.47 & 75.77 & 2.85 \\
90 & 76.25 & 3.42 & 496.07 & 2.76 \\
{$[0 / 45 / 90 /-45]_{2 \mathrm{~S}}$} & 490.33 & 24.08 & 23.37 \\
\hline
\end{tabular}




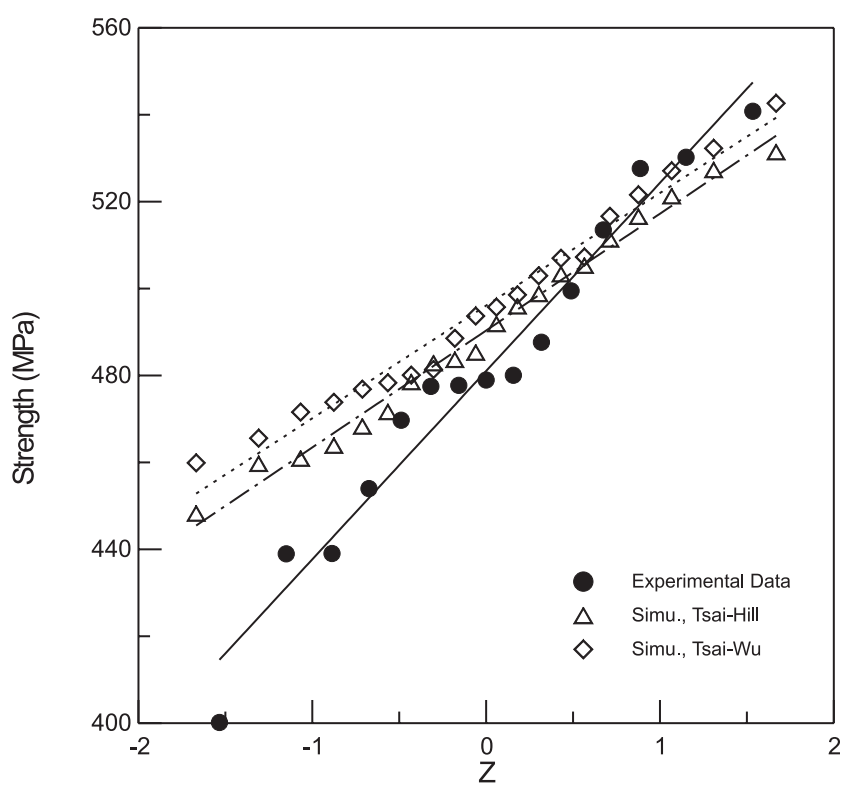

Fig. 6. Strengths of $[0 / 45 / 90-45]_{2 s}$ laminates plotted in normal probability paper.

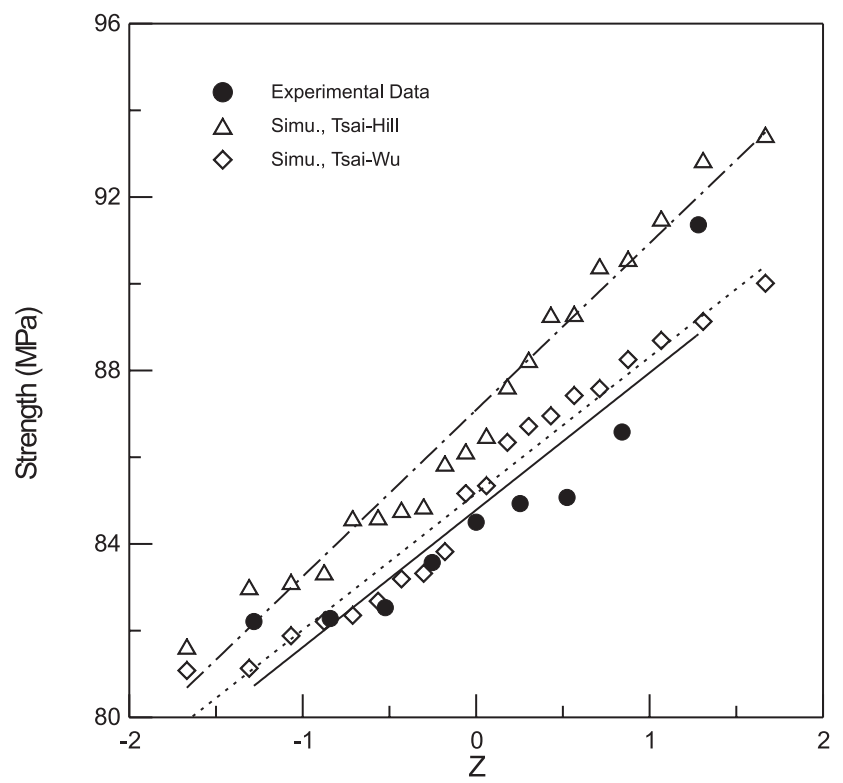

Fig. 7. Strengths of $60^{\circ}$ laminates plotted in normal probability paper.

characterize the tensile strengths of the composite laminates studied. This information is useful for a design engineer.

\section{Acknowledgements}

This work was supported by the National Science Council of the Republic of China under Grant No. NSC

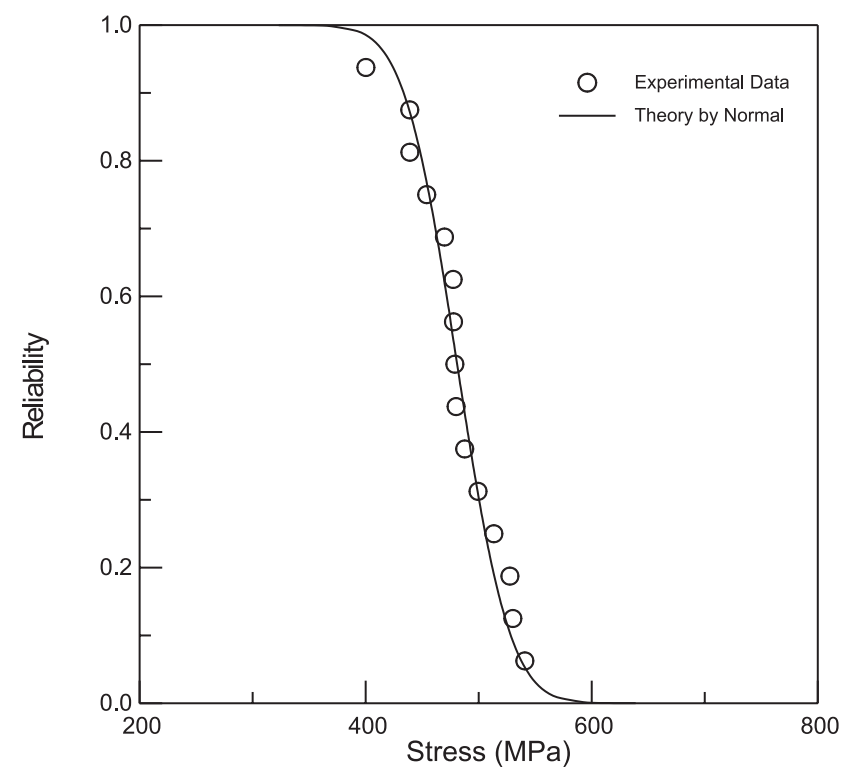

Fig. 8. Reliability of $[0 / 45 / 90-45]_{2 s}$ laminates when subjected to unidirectional stress.

85-2212-E-002-037. The writers are grateful for this support.

\section{References}

[1] Cassenti BN. Probabilistic static failure of composite materials. AIAA J 1984;22:103-10.

[2] Wang ASD, Chou PC, Lei SC. A stochastic model for the growth of matrix cracks in composite laminates. J Composite Mater 1984;18:239-54.

[3] Yang JN, Yang SH, Jones DL. A stiffness-based statistical model for predicting fatigue life of graphite/epoxy laminates. J Composite Technol Res, ASTM 1989;11:129-34.

[4] Zhu YT, Zong G. On the application of the statistical strength model of fiber-reinforced composites. J Composite Mater 1993;27:944-59.

[5] Wetherhold RC, Ucci AM. Probability methods for the fracture of composite materials. Composite Struct 1994;28:113-9.

[6] Goda K, Phoenix SL. Reliability approach to the tensile strength of unidirectional CFRP composites by Monte-Carlo simulation in a shear-lag model. Composite Sci Tech 1994;50:457-68.

[7] Kam TY, Chang ES. Reliability formulation for composite laminates subjected to first-ply failure. Composite Struct 1997;38:447-52.

[8] Jeong HK, Shenoi RA. Reliability analysis of mid-plane symmetric laminated plates using direct simulation method. Composite Struct 1998;43:1-13.

[9] Vanmarcke EH. Random fields. Cambridge, MA, USA: IT Press, 1983.

[10] Shinozuka M. Stochastic mechanics, vol. III. Technical Report, Department of Civil Engineering and Operations Research, Princeton University, Princeton, NJ, USA, 1988.

[11] Ang AH-S, Tang WH. Probability concepts in engineering planning and design, vol. II. New York, USA: Wiley, 1984.

[12] Jones RM. Mechanics of composite materials. Washington, DC, USA: Scripta Book Company, 1975.

[13] Tsai SW, Hahn HT. Introduction to composite materials. Lancaster, PA, USA: Technomic, 1980. 
[14] Gibson RF. Principles of composite material mechanics. New York, USA: McGraw-Hill, 1994.

[15] Hyer MW. Stress analysis of fiber-reinforced composite materials. Boston, USA: WCB/McGraw-Hill, 1998.

[16] Shinozuka M. Stochastic fields and their digital simulation. In: Schueller GI, Shinozuka M. editors. Stochastic methods in structural dynamics. Dordrecht, The Netherlands: Martinus Nijhoff, 1987: 93-133.

[17] Halpin JC. Primer on composite materials: analysis. Lancaster, PA, USA: Technomic, 1984.

[18] Ang AH-S, Tang WH. Probability concepts in engineering planning and design, vol. I. New York, USA:Wiley, 1975. 